\title{
Complexity/informativeness trade-off in the domain of indefinite pronouns*
}

\author{
Milica Denić \\ University of Amsterdam \\ Shane Steinert-Threlkeld \\ University of Washington at Seattle \\ Jakub Szymanik \\ University of Amsterdam
}

\begin{abstract}
The vocabulary of human languages has been argued to support efficient communication by optimizing the trade-off between complexity and informativeness (Kemp \& Regier 2012). The argument has been based on cross-linguistic analyses of vocabulary in semantic domains of content words such as kinship, color, and number terms. The present work extends this analysis to a category of function words: indefinite pronouns (e.g. someone, anyone, no-one, cf. Haspelmath 2001). We build on previous work to establish the meaning space and featural make-up for indefinite pronouns, and show that indefinite pronoun systems across languages optimize the complexity/informativeness trade-off. This demonstrates that pressures for efficient communication shape both content and function word categories, thus tying in with the conclusions of recent work on quantifiers by Steinert-Threlkeld (2019). Furthermore, we argue that the trade-off may explain some of the universal properties of indefinite pronouns, thus reducing the explanatory load for linguistic theories.
\end{abstract}

Keywords: indefinites; complexity; informativeness; trade-off; efficiency; linguistic universals; function words

\section{Introduction}

The vocabulary of human languages has been argued to support efficient communication by optimizing the trade-off between complexity and informativeness (Kemp \& Regier 2012). In informal terms, the complexity of a system is a measure of how

* We wish to thank Lisa Bylinina, Fausto Carcassi, Gennaro Chierchia, Mora Maldonado, and Terry Regier, as well as the audiences of the MLC seminar at the University of Amsterdam, the CLE seminar at the University of Edinburgh, CogSci 2020 and SALT30 for helpful discussions. The research leading to these results has received funding from the European Research Council under the European Union's Seventh Framework Programme (FP/2007- 2013) / ERC Grant Agreement n. STG $716230 \mathrm{CoSaQ}$. 
easy it is to mentally represent that system. The informativeness of a system is a measure of how precisely the system allows us to communicate intended meanings. These two properties of a system trade-off against each other (Zipf 1949; Ferrer i Cancho \& Solé 2003; Rosch 1978; Kemp \& Regier 2012). The reason is that, in general, the fewer expressions a language has, the fewer semantic distinctions it is able to make, but the easier it will be to mentally represent. In other words, simplifying the language often entails sacrificing informativeness, and improving informativeness often requires added complexity. That the category systems of natural languages are (near-)optimal solutions to trading off these two measures has been argued based on cross-linguistic data and generalizations coming from various semantic domains: kinship terms, color terms, container terms, number terms, and more recently quantifiers and connectives (Kemp \& Regier 2012; Regier, Kemp \& Kay 2015; Xu \& Regier 2014; Xu, Regier \& Malt 2016; Steinert-Threlkeld 2019; Uegaki 2020; Xu, Liu \& Regier 2020).

The present work extends this analysis to indefinite pronouns, a domain of function words whose syntactic, semantic and typological properties have been extensively studied by comparative linguists (Haspelmath 2001). Examples of indefinite pronouns in English are expressions such as someone, something, anyone, anything, no-one, nothing. ${ }^{1}$

There are at least two reasons to pursue the extension of this framework to indefinite pronouns. First, it would strengthen the case that the complexity/informativeness trade-off shapes both content and function word categories in language. Recent work has provided some evidence in this direction in the case of quantifiers and connectives (Steinert-Threlkeld 2019; Uegaki 2020). However, the lack of an appropriate cross-linguistic dataset on quantifiers makes it impossible at the moment to categorically defend the claim that the category of quantifiers in natural languages is optimized for complexity/informativeness trade-off. The case of indefinite pronoun systems helpfully differs because such a rich cross-linguistic dataset is available in this domain: Haspelmath's (2001) seminal work on indefinite pronouns includes a dataset on their meaning and distribution in 40 languages. We will rely heavily on this data in conducting the aforementioned efficiency analyses.

Second, this analysis would help delineate which properties of indefinite pronoun systems cross-linguistically follow from more general communication pressures, and which need to be captured by independent (syntactic or semantic) theories.

1 We note that the term indefinite pronoun is not a standard term for these expressions in the formal semantics literature. However, as Haspelmath's (2001) work on these expressions is well known and widely cited, we have opted for keeping the term. Roughly, in more standard terminology, the expressions categorized as indefinite pronouns by Haspelmath (2001) include "vanilla" existential indefinites, negative polarity indefinites, epistemic indefinites, free choice indefinites, and negative indefinites (including negative concord indefinites and negative quantifiers). 
More concretely, a striking finding of Haspelmath's typological research is that no two languages in his corpus have the same systems of indefinite pronouns: in other words, it is not possible to establish a one-to-one mapping between any two languages' indefinite pronouns' meaning and distribution. This diversity is however constrained in important ways, which Haspelmath formulates as implicational linguistic universals. Evaluating whether indefinite pronoun systems optimize the complexity/informativeness trade-off can thus help make progress on the following question: why do indefinite pronoun systems vary across languages, and why is this variation constrained in the way it is?

The paper is organized as follows. First, we set the background for our analysis by (i) describing the meaning space of indefinite pronouns, (ii) detailing how Haspelmath's (2001) dataset is used in our research, and (iii) explaining how informativeness and complexity of languages are measured. We then report the results of two computational experiments. The first experiment demonstrates that natural languages' indefinite pronoun systems are (near-)optimal in how they trade off complexity and informativeness. The second experiment demonstrates that Haspelmath's implicational universals play a role in the complexity/informativeness trade-off optimization. Finally, we discuss the implications of the results in light of assumptions on which this type of analysis is founded.

\section{Meaning space of indefinite pronouns}

We first explain the space of possible meanings expressed by indefinites: what are the meanings that interlocutors may want to communicate by an indefinite pronoun?

Haspelmath (2001) describes each indefinite pronoun in each language in his dataset in terms of which functions it can take. These functions are depicted on a "map" in Figure 1. Some of them are meaning-driven (functions 1, 2, 3, 9 in Figure 1, i.e. specific known, specific unknown, non-specific, free choice), and others are driven by syntactic distribution (functions 4, 5, 6, 7, 8, i.e. question, conditional, comparative, indirect negation, direct negation in Figure 1). 


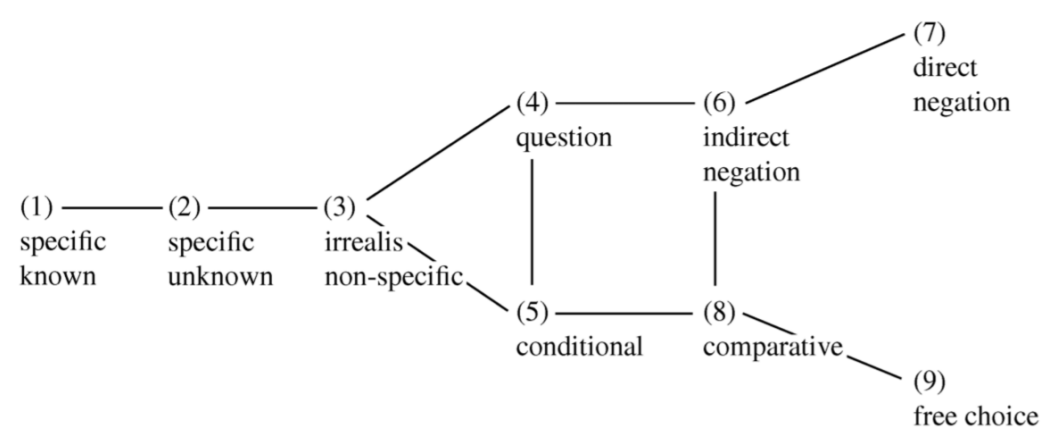

Figure 1 Haspelmath's map of functions of indefinites.

Haspelmath's functions thus mix meaning and syntactic distribution, and as such cannot be (all) taken as components of the meaning space of indefinite pronouns. To focus purely on meaning, we introduce semantic flavors and translate Haspelmath's syntactic functions into them. More specifically, Haspelmath's syntactic functions relate to two well-studied categories of indefinite pronouns: negative polarity indefinites and negative indefinites (Fauconnier 1975; Ladusaw 1979; Haspelmath 2001; Penka 2011; Bernini \& Ramat 1996). In the present work, we abstract away from certain differences in the syntactic distribution of different negative polarity indefinites, and likewise, of different negative indefinites. We thus assume that the meaning space that the system of indefinite pronouns has to cover consists of the following six 'semantic flavors', which are described informally and illustrated with an example in (1)-(6).

(1) Specific known flavor [the indefinite pronoun refers to a specific individual that the interlocutors can uniquely identify]:

Someone managed to mess this up — we all know who!

(2) Specific unknown flavor [the indefinite pronoun refers to a specific individual that the interlocutors cannot uniquely identify]:

I heard that someone failed, but I don't know who.

(3) Non-specific flavor [the indefinite pronoun is interpreted as an existential quantifier over some domain of possible referents, not referring to a specific individual]:

You should probably talk to someone else about this too.

(4) Negative polarity flavor [the indefinite pronoun is interpreted as an existential quantifier over a widened domain of possible referents]:

Less than three companies hired anyone this year.

(5) Free choice flavor [the indefinite pronoun is interpreted as a wide-scope 
Denić, Steinert-Threlkeld and Szymanik

universal quantifier over some domain of possible referents]: You can hire almost anyone here: most of them great.

(6) Negative indefinite flavor [the indefinite pronoun is interpreted as a negated existential quantifier over some domain of possible referents]:

Who went to the party? No one.

We indexed each indefinite pronoun in Haspelmath's (2001) corpus with the flavors it can convey as follows.

- Functions 1, 2, 3, 9 correspond to flavors 1, 2, 3, 5 respectively.

- To decide whether an indefinite pronoun can convey flavor 6, we collected data on whether indefinite pronouns in languages of Haspelmath's corpus can be interpreted as negated existentials, relying in most cases on occurrence in negative fragment answers, which is a test for negative indefinites (Bernini \& Ramat 1996). ${ }^{2}$

- If (a) an item can take at least one of the functions 4 and 6 but not function 3 ; or (b) it can take function 8 and at least one of the functions 4 and 6 , or it can take function 8 but cannot take function 9; or (c) it can take function 7 but not function 3 and cannot be interpreted as a negated existential; or (d) it can take function 5 , but not functions 3 and 9 , then it has flavor $4 .^{3}$

2 These data are available in an online Appendix to the paper at https://github.com/milicaden/indefinitepronouns-SALT.

3 The motivation behind this complicated disjunctive criterion for negative polarity flavor is as follows. (a) Both indefinites with non-specific flavor and indefinites with negative polarity flavor can be used in questions and under the scope of negation, hence we can only conclude from functions 4 and 6 that an indefinite has a negative polarity flavor if we know independently that it cannot get non-specific flavor. (b) Indefinite pronouns with function 8 might have either negative polarity or free choice flavor: items with negative polarity but not free choice flavor such as English ever are acceptable in comparatives, but so are instances of any modified by almost, and modification by almost is commonly taken as evidence for the free choice interpretation of any (cf. Aloni \& Roelofsen 2014; Heim 2006). If an item can take function 8 in combination with functions 4 and/or 6 which code for the negative polarity environments (any cannot be modified by almost in those environments which evidences that free choice flavor is not available in those environments), we may conclude that the indefinite pronoun can have the negative polarity flavor. Similarly, if an indefinite pronoun takes function 8 but not function 9, we may conclude that it cannot convey the free choice flavor and thus must be conveying the negative polarity flavor. (c) As all negative indefinites are indexed with function 7 by Haspelmath, we only rely on function 7 as revealing negative polarity flavor if the indefinite in question cannot be interpreted as negated existential (and if it cannot get non-specific flavor more generally, for the same reasons as above). (d) Finally, in the antecedent of conditionals we may find indefinites with negative polarity, non-specific, and free choice flavor; we can thus conclude from function 5 that an indefinite has a negative polarity flavor if we know independently that it cannot get non-specific or free choice flavor. 
Complexity/informativeness trade-off in the domain of indefinite pronouns

The data on indefinite pronouns presented in Haspelmath 2001 shows that languages differ greatly in how they cover the meaning space with their lexical items. Haspelmath however established that this variation is constrained in the following way: any indefinite pronoun in any language can only take functions which form a connected area on the map in Figure 1. For instance, if an indefinite pronoun can take functions 2 and 4, it is also able to take function 3 . It is relatively straightforward to 'translate' Haspelmath's universals from functions to flavors in the same principled way as established above. Here are two examples of such 'translations' of universals. (i) If an item can convey the specific unknown and the negative polarity flavor, it can convey the non-specific flavor. (ii) If an item can convey the specific known and the non-specific flavor, it can also convey the specific unknown flavor.

\section{Measuring complexity and informativeness}

\subsection{Complexity}

Our measure of complexity relies on featural make-up of indefinite pronouns. For this measure, we will again build on Haspelmath's work. Haspelmath 2001: §5 proposes that there are 5 binary features indefinite items can carry: known to the speaker $(K)$, specific $(S)$, scalar endpoint $(S E)$, scale reversal $(R)$, and in the scope of negation $(N)$. Haspelmath further assumes that the feature $R(+$ or - ) requires the indefinite pronoun to carry $S E+$ feature.

Let us review briefly what these features stand for in Haspelmath's work. The features $K$ and $S$ are relatively transparent. $S$ relates to the semantic notion of specificity, i.e. to whether the speaker has a specific referent in mind for the indefinite pronoun. $K$ relates to whether or not the referent is known to the speaker. As for $S E$, Haspelmath 2001: $\$ 5$ motivates it from Fauconnier's work on negative polarity and free choice indefinites: in short, negative polarity and free choice indefinites evoke a pragmatic scale of alternatives ordered by likelihood, and they associate with its lowest (least likely) endpoint (Fauconnier 1975). Scale reversing contexts reverse the order of alternatives on the pragmatic scale; these are essentially downwardentailing contexts. $R$ feature reflects that negative polarity indefinites should associate with the lowest point on the scale in the scale reversing contexts $(R+)$, while free choice indefinites associate with the lowest point on the scale in the non-scale reversing contexts $(R-)$. Finally, $N$ feature relates to whether the indefinite pronoun necessarily appears in the scope of negation.

Haspelmath assumes that each of these five binary features characterizes a subset of functions of indefinite pronouns (cf. Haspelmath 2001: $\$ 5$ for details). In the continuation, we assume the five binary features to characterize various semantic 
Denić, Steinert-Threlkeld and Szymanik

flavors as follows ${ }^{4}$ :

- $K+$ : specific known

$K-$ : specific unknown, non-specific, negative polarity, free choice, negative indefinite

- $S+$ : specific known, specific unknown

$S-$ : non-specific, negative polarity, free choice, negative indefinite

- $S E+$ : negative polarity, free choice, negative indefinite

$S E-$ : specific known, specific unknown, non-specific

- $R+:$ negative polarity, negative indefinite

$R-:$ free choice

- $N+$ : negative indefinite

$N-$ : specific known, specific unknown, non-specific, negative polarity, free choice

Haspelmath does not provide a general recipe for how to generate the featural make-up of an indefinite from the combination of functions that it might be able to take. We provide here one such recipe which is simple yet general enough to enable us to define any item in terms of its feature content based on the semantic flavors it can convey. Let us treat features as sets of flavors that they characterize, and the combination of features to correspond to set-theoretic operations of intersection or union. We then define the featural make-up of the indefinite pronoun to be the shortest formula - in a language whose primitives are the five binary features listed above and the set-theoretic operations of union and intersection - that would correspond exactly to the flavor(s) an item can convey. For instance, in this language, the two formulae ' $K+$ ' and ' $K+\cap S+$ ' amount to the same set of flavors, i.e. \{specific known . However, because ' $K+$ ' is a shorter formula than ' $K+\cap S+$ ', we consider the featural make-up of an indefinite pronoun that conveys only the specific known flavor to be ' $K+$ ' and not ' $K+\cap S+$ '.

4 The features $S E+$ and $R+$ are considered to characterize the negative indefinite flavor for two reasons. First, Haspelmath considers these two features to characterize the direct negation function, and as discussed, all indefinite pronouns with the negative indefinite flavor are indexed by Haspelmath as having the direct negation function. Second, these two features characterize the negative polarity flavor, and at least certain negative indefinites (the so-called negative concord items or N-words) have been argued to simply be a special type of negative polarity indefinites (i.e. negative polarity items which can induce the presence of negation); see for instance Chierchia 2013.

5 It is worthwhile pointing out that the features Haspelmath proposes for indefinite pronouns are clearly applicable to items from various other domains. For instance, the concept of scale has been argued to play a role in the semantics of scalar particle even (Karttunen \& Peters 1979), and linguists have in 
Complexity/informativeness trade-off in the domain of indefinite pronouns

We measure the complexity of an item $c(i)$ as the number of features in its featural make-up (repetitions of the same features are counted as well). For instance, the featural make-up of an item which can convey only the specific unknown flavor would be ' $S+\cap K-$ ', and its complexity would thus be 2 . Our measure of complexity of a language $\operatorname{Comp}(L)$ is defined as the sum of complexity measures of each item in the language (cf. (7)).

\section{Complexity of a language:}

$$
\operatorname{Comp}(L)=\sum_{i \in L} c(i)
$$

\subsection{Informativeness}

Our measure of informativeness is rooted in the notion of successful communication: a speaker has a semantic flavor in mind that they want to communicate to a listener by using an indefinite pronoun in their language (Skyrms 2010; Kemp, Xu \& Regier 2018; Steinert-Threlkeld 2019). It corresponds to the probability that the communication will be successful given the prior over flavors from the set of flavors $F$, the conditional probability $P(i \mid f)$ which reflects the probability that the speaker uses the indefinite pronoun $i$ to communicate $f$, and $P(f \mid i)$ the probability that the listener correctly guesses $f$ upon hearing $i$ (cf. (8)).

\section{Informativeness of a language:}

$$
I(L)=\sum_{f \in F} \sum_{i \in L} P(f) P(f \mid i) P(i \mid f)
$$

The prior over flavors is estimated from the corpus in Beekhuizen, Watson \& Stevenson 2017 in which indefinite pronouns are annotated for functions. Going from functions to semantic flavors, ${ }^{6}$ estimated priors over flavors are provided in Table 1.

fact drawn connections between scalar particles and negative polarity indefinites (e.g. Lee \& Horn 1994).

6 This was done using the English version of the corpus in Beekhuizen et al. 2017 as follows. If an indefinite pronoun was anyone or anybody and it was annotated to have one of the functions question, indirect negation, direct negation, we indexed it as having the negative polarity flavor. If an indefinite pronoun was anyone or anybody and it was annotated to have one of the functions conditional, comparative, we indexed it as having the negative polarity flavor half of the time, and as having the free choice flavor half of the time (this is because these environments allow for both negative polarity and free choice flavor; we have no data on the rate of each of these flavors in these two environments and thus assume them to be equally frequent). If an indefinite pronoun was someone, somebody and it was annotated to have one of the functions question, indirect negation, direct 
Denić, Steinert-Threlkeld and Szymanik

\begin{tabular}{ll}
\hline Semantic flavor & Prior probability \\
\hline specific known & 0.08 \\
specific unknown & 0.08 \\
non-specific & 0.26 \\
negative polarity & 0.33 \\
free choice & 0.1 \\
negative indefinite & 0.15 \\
\hline
\end{tabular}

Table 1 Prior probability distribution over flavors, as estimated from the corpus in Beekhuizen et al. 2017.

The conditional distributions are assumed to be uniform: $P(i \mid f)=\frac{1}{n}$ if $f \in i$, 0 otherwise, where $n=|\{i: f \in i\}| ; \quad P(f \mid i)=\frac{1}{n}$ if $f \in i, 0$ otherwise, where $n=|\{f: f \in i\}|$. The fact that conditional distributions are assumed to be uniform entails that the communicative scenario being modeled is that between a 'literal speaker' and a 'literal listener' (cf. Frank \& Goodman 2012).

The communicative cost of a language should be a decreasing function of the informativeness of the language; we define the communicative cost of a language $L$ in (9). This means that maximizing informativeness of a language is equivalent to minimizing communicative cost; we will use these two terms interchangeably.

(9) Communicative cost of a language:

$$
\operatorname{Cost}(L)=\frac{1}{I(L)}
$$

\section{Computational experiment 1}

In Experiment $1^{7}$, we address the question of whether natural languages optimize the complexity/informativeness trade-off in the domain of indefinite pronouns.

negation, conditional, comparative, we indexed it as having the non-specific flavor. If an indefinite pronoun was no one, nobody, we indexed it as having the negative indefinite flavor. Indefinite pronouns annotated with functions non-specific and free choice were indexed as having corresponding semantic flavors. Beekhuizen et al. (2017) did not distinguish between specific known and specific unknown functions, and annotated the indefinite pronouns which had one of those two functions with specific only. For lack of evidence to the contrary, we assume that among the indefinite pronouns annotated with specific by Beekhuizen et al. (2017), those with specific known and those with specific unknown flavor are equally frequent.

7 The scripts used for Experiments 1 and 2 can be found at https://github.com/milicaden/indefinitepronouns-SALT. 
Complexity/informativeness trade-off in the domain of indefinite pronouns

For our purposes, a language is a set of indefinite pronouns for the ontological category PERSON (e.g. someone, anyone, no one). ${ }^{8}$ We evaluate the optimality of natural languages by measuring how distant they are from the Pareto frontier in comparison to artificial languages. The Pareto frontier consists of Pareto optimal languages; a language is (Pareto) optimal if there is no other language that has both lower complexity and lower communicative cost. The idea is that, if natural languages optimize complexity/informativeness trade-off, they should be more optimal than randomly generated languages, and being more optimal means being closer to the set of optimal languages (the Pareto frontier).

We computed complexity and communicative cost measures for each of the 40 languages in Haspelmath's corpus. We artificially generated 10000 languages, which could have between 1 and 7 indefinite pronouns ( 7 is the maximum number of indefinite pronouns that any natural language has in Haspelmath's corpus). Each indefinite pronoun in each artificial language was randomly assigned one of the 63 logically possible combinations of flavors $\left(2^{6}-1\right.$ combination whereby an indefinite pronoun doesn't convey any of the 6 flavors).

The artificial languages were then matched to natural languages for the degree of synonymy, defined as in (10). The degree of synonymy captures how many different indefinites can be used to express a flavor: if the indefinites in a language have more overlapping meanings, the degree will be higher. Matching ensured that for each degree of synonymy $d$ of natural languages, the proportion of artificial languages with $d$ was the same as the proportion of natural languages with $d$. After matching, 2133 artificial languages remained for comparison to natural languages (mean degree of synonymy in both groups is 0.67 ).

\section{Degree of synonymy:}

$$
\operatorname{Syn}(L)=\sum_{f \in F}|\{i \in L: f \in i\}|-1
$$

These artificially generated languages serve to map the space of possibilities for indefinite pronoun systems whose number of words and degree of synonymy are comparable to that of natural languages. We plot complexity and communicative cost of each of the natural and artificial languages in Figure 2.

We follow Steinert-Threlkeld (2019) in using an evolutionary algorithm to estimate the Pareto frontier. The algorithm works as follows. First, the generation 0 is generated, which consists of 2000 randomly generated languages. The dominant

8 Comparable experiments may be conducted for other ontological categories, such as THING, TIME, PLACE, etc. What may change in these additional experiments are the prior probability distributions over flavors and the inventory of indefinite pronouns for natural languages, as natural languages may have gaps in their indefinite pronoun paradigms for certain ontological categories. 
languages (those for which there is no language which has both lower complexity and lower communicative cost) each give rise to an equal number of offspring languages, which are obtained via a small number of mutations (between 1 and 3; these mutations included removing an item, adding an item, and interchanging an item) from dominant languages. The dominant languages from generation 0 together with their offspring languages constitute generation 1. This process is repeated for 100 generations. Finally, the dominant languages are selected from the union of the last generation, the 40 natural languages, and the 10000 artificial languages generated for Experiment 1. A curve in (11) is then fitted to the dominant languages to estimate the Pareto frontier. The estimated Pareto frontier is plotted as the black curve in Figure 2.

$$
\operatorname{Comp}(L) \sim \frac{a}{b+\operatorname{Cost}(L)}
$$

As can be seen in Figure 2, most of the 40 natural languages lie near this Pareto frontier, suggesting that they are optimizing complexity/informativeness trade-off. This is further supported by the comparison of distances from the Pareto frontier of natural and artificial languages. For each language, we compute its distance from the Pareto frontier as the minimum Euclidean distance between the language and a point on the Pareto frontier. We find that natural languages are significantly closer to the Pareto frontier than artificial languages $\left(M_{1}=0.55, M_{2}=2.21, t(55.5)=-27.2\right.$, $p<.001)$. Interestingly, we see that while most natural languages lie in the lower left corner of the plot, with low communicative cost and low complexity, there is quite some variability in terms of what the closest point on the Pareto frontier for each of the natural languages would be. This suggests that some of the diversity that can be observed in the indefinite pronoun systems across languages is due to languages approaching different optimal solutions to the trade-off problem. 
Complexity/informativeness trade-off in the domain of indefinite pronouns

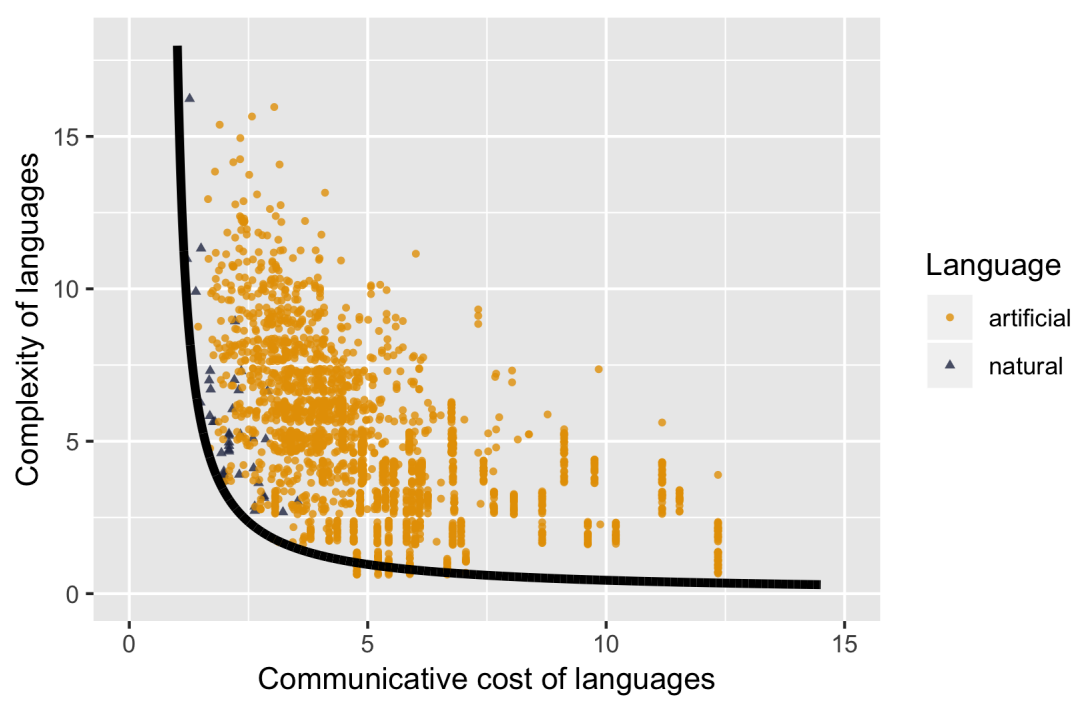

Figure 2 Experiment 1: Complexity and communicative cost of 40 natural and 2133 artificial languages (natural and artificial languages matched for the degree of synonymy).

\section{Computational experiment 2}

Experiment 1 demonstrates that natural languages optimize the complexity/informativeness trade-off of their indefinite pronoun systems. The trade-off optimization is thus likely to explain some of the variation between indefinite pronoun systems among natural languages, as well as some of their universal properties. Does the trade-off explain some of the Haspelmath's universals? To answer this question, in Experiment 2, we compare 5000 artificial languages which satisfy Haspelmath's universals, as originally stated, in terms of functions rather than flavors, ${ }^{9}$ to 5000 artificial languages which do not (henceforth Haspel-ok and Not Haspel-ok languages respectively). We do this comparison for the following reason: if the reason why natural languages satisfy Haspelmath's universals is because these help optimize complexity/informativeness trade-off, then artificial languages which satisfy Haspelmath's universals should be more optimal than artificial languages which do not satisfy them. Languages of both groups had between 1 and 7 items, and they were

9 Recall that all items which convey the negative indefinite flavor, such as English no one, are indexed as having direct negation function by Haspelmath (but they are not the only items to be indexed as having direct negation function). As we relied on acceptability in negative fragment answers to be the distinguishing test for the negative indefinite flavor, we consider the negative fragment answer to be the 10th function, and add an extra universal to Haspelmath's list: if an indefinite pronoun can have the negative fragment answer function, it can also have the direct negation function. 


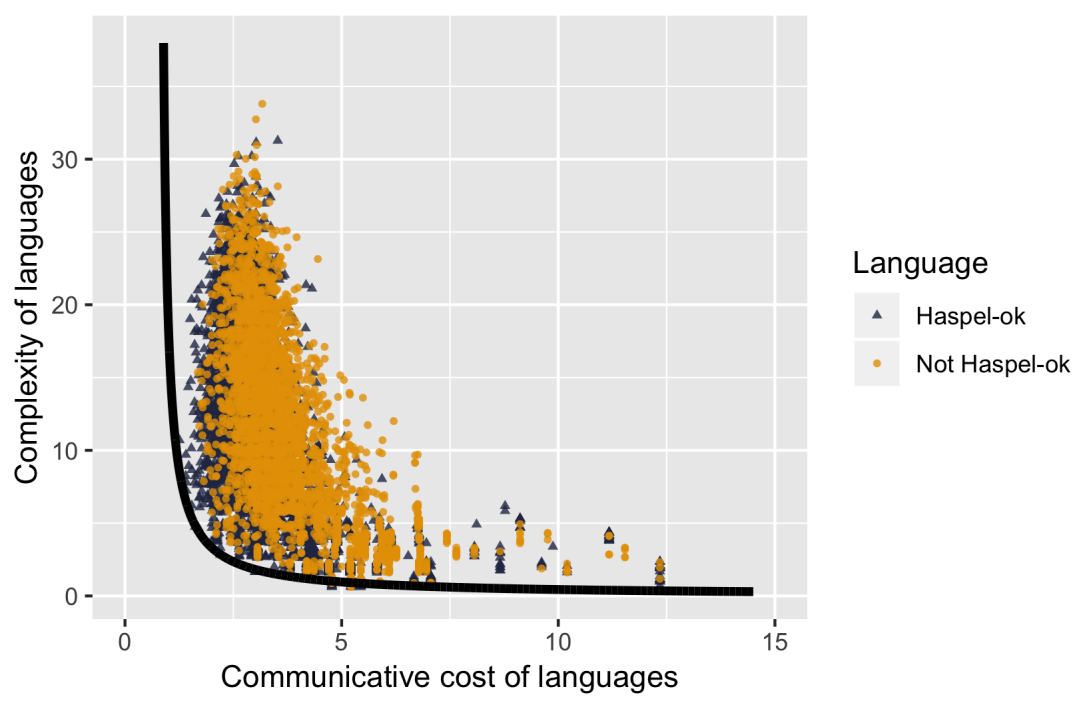

Figure 3 Experiment 2: Complexity and communicative cost of 5000 artificial languages which satisfy Haspelmath's universals and 3281 artificial languages which do not (Haspel-ok and Not Haspel-ok languages matched for the degree of synonymy).

matched for their degree of synonymy (after matching, all 5000 Haspel-ok languages and 3281 Not Haspel-ok languages remain, with mean degree of synonymy in both groups 5.6). Each item in each Haspel-ok language is sampled from a pool of all logically possible items which satisfy Haspelmath's universals (in terms of which of Haspelmath's functions they can take). On the other hand, each item in each Not Haspel-ok language is samped from a pool of all logically possible items (in terms of which of Haspelmath's functions they can take): items of Not Haspel-ok languages thus may or may not conform to Haspelmath's universals. The Pareto frontier is estimated in the same way as in Experiment 1.

In Figure 3, we plot the complexity and communicative cost of Haspel-ok and of Not Haspel-ok languages, as well as the estimated Pareto frontier. Using the same measure of distance from Pareto frontier as in Experiment 1, we find that the Haspel-ok languages are significantly closer to Pareto frontier than the Not Haspel-ok languages $\left(M_{1}=1.7, M_{2}=2.22, t(6242.3)=-31.1, p<.001\right)$. This demonstrates that languages which satisfy Haspelmath's universals are indeed better at trading complexity and informativeness than languages which do not. Importantly, the results of Experiment 2 demonstrate that this holds in general, and not only for the 40 natural languages from Haspelmath's corpus. 
Complexity/informativeness trade-off in the domain of indefinite pronouns

\section{Discussion}

In this work, we have argued that the complexity/informativeness trade-off can explain how a language organizes its vocabulary to cover the meaning space expressed by indefinite pronouns. In general, such work relies on three fundamental assumptions: (1) that we are indeed dealing with a proper category (i.e. that all and only the expressions we are considering form a (sub-)system of a language); (2) that we have a good understanding of the meaning space that the system is covering; (3) that we have a reasonable way to estimate the complexity and informativeness of the system. These assumptions are sometimes left implicit in related work; we discuss them explicitly in relation to indefinite pronouns, and point out where they may be questioned and may evolve. We believe that the points we raise in this discussion may be of relevance for any work belonging to this paradigm.

\subsection{Are 'indefinite pronouns' a well-defined category?}

The question of whether the system of indefinite pronouns is optimized with respect to the complexity/informativeness trade-off presupposes that the expressions that fall under the label 'indefinite pronoun' indeed form a category across languages. One way to establish that something is a category is to provide a set of criteria that would separate members from non-members. Haspelmath (2001) defines indefinite pronouns as expressions (i) which are grammatical, i.e. function words, that are syntactically mainly noun phrases, adverbial phrases, or adjectival phrases, and (ii) whose main semantic function is to express indefinite reference. Haspelmath however acknowledges that (ii) is too narrow to qualify as indefinite pronouns all and only the expressions that he lists under the term: for instance, the indefinite pronoun nobody arguably does not express indefinite reference, but rather conveys the non-existence of a referent. A more general semantic criterion is thus needed for the category of indefinite pronouns to be well-defined.

While it is beyond the scope of this work to attempt to identify such a more general semantic criterion, let us point out that much semantic work since Haspelmath has indeed argued in favor of close semantic connections between expressions that Haspelmath considers to be 'indefinite pronouns'. For instance, Chierchia (2013) argues that expressions such as someone, something and anyone, anything and their cross-linguistic counterparts have a common semantic core; much work assumes that expressions such as no-one, nothing are underlyingly negation merged with an indefinite expression such as someone, something (cf. Jacobs 1980 and much subsequent work). Furthermore, the expressions that Haspelmath subsumes under the term 'indefinite pronouns' are often diachronically related across languages, suggesting that their meanings are closely related (Chierchia 2013; Roberts \& Rous- 
sou 2003; Jäger 2010). While we can thus have some confidence in the reality of the category of indefinite pronouns, the task of justifying the categories posited must be addressed in any work of this kind. This task becomes more pressing in domains of functional vocabulary. In content word domains (e.g. color terms), one can use criteria about the kinds of entities referred to by the expression (e.g. colors) to demarcate the sub-system; but functional domains have more abstract meaning spaces, which makes these criteria harder to apply in practice.

\subsection{Meaning space of indefinite pronouns}

Investigating how efficiently systems of indefinite pronouns cover a certain meaning space presupposes that we have a good understanding of what the meaning space consists of.

We have described in Section 2 the six semantic flavors assumed to constitute the meaning space of indefinite pronouns. As indicated there, however, in doing so we have abstracted away from a number of subtle differences in syntactic distributionespecially in the domain of items with negative polarity and negative indefinite flavor-which may very well reflect subtle meaning differences. To illustrate the complexity of the situation, among negative polarity indefinites, there is a bewildering diversity in syntactic and semantic behavior of various expressions (cf. for instance the literature on strong, weak, and Bagel problem negative polarity items (Krifka 1994; Pereltsvaig 2004: a.o.)). The situation is similar in the domain of free choice and negative indefinites (Chierchia 2013; Zeijlstra 2004). We acknowledge that more work is needed to better understand the semantic correlates of these various types of negative polarity, free choice and negative indefinites, which may call for revising the meaning space we have assumed.

\subsection{Measures of complexity and informativeness of languages}

To construct a complexity measure for the systems of indefinite pronouns, we have built on the theory of features of indefinite pronouns put forward in Haspelmath 2001. To our knowledge, Haspelmath's is the only feature-based theory intended to account for all of the semantic flavors of indefinite pronouns discussed here. There are, however, more recent approaches to the feature content of some of the subcategories of indefinite pronouns, such as negative polarity and free choice indefinites (see for instance Chierchia 2013). These recent proposals may be developed further to construct alternative measures of complexity. In addition to this, one can conceive of measures of complexity not based on features, but for instance, solely on the number of items in the system.

Similarly, one may consider alternative measures of informativeness. Recall 
Complexity/informativeness trade-off in the domain of indefinite pronouns

that according to the measure of informativeness we employed, the communicative scenario being modeled is that between a 'literal speaker' and a 'literal listener' (cf. Section 3.2). We note that other options are conceivable here: for instance, the communicative scenario may be that between a 'literal listener' and a 'pragmatic speaker', or a 'pragmatic listener' and a 'literal speaker', etc. (cf. Frank \& Goodman 2012 and other work in the Rational Speech Act framework tradition).

It would be interesting to explore how well our results generalize to these alternative measures of complexity and informativeness. We leave this exploration for future work.

\section{Conclusions}

We find that natural languages optimize the complexity/informativeness trade-off in how they organize their indefinite pronoun systems. These results represent an extension of efficiency analyses to a system of function words, thus tying in with Steinert-Threlkeld 2019 in concluding that similar communication pressures are shaping both content and function word categories across languages.

Focusing on the implications of our results for the study of indefinite pronouns, we have proposed that some of the diversity we observe among languages is due to different optimal solutions to the complexity/informativeness trade-off. The complexity/informativeness trade-off may also explain some universal properties of indefinite pronoun systems: we find that Haspelmath's universals contribute to the complexity/informativeness trade-off optimization. A question that remains for future work is to find out which of Haspelmath's universals help with the optimization: it is conceivable that only a proper subset of them do, and that the rest need a different explanation.

Finally, we have discussed the assumptions required at each step of this efficiency analysis in the case of indefinite pronouns. These considerations must be made in any such analysis, and a thorough discussion of the choice points can help illuminate future work in this and related domains.

\section{References}

Aloni, Maria \& Floris Roelofsen. 2014. Indefinites in comparatives. Natural Language Semantics 22(2). 145-167. doi:https://doi.org/10.1007/s11050-0139103-z.

Beekhuizen, Barend, Julia Watson \& Suzanne Stevenson. 2017. Semantic typology and parallel corpora: Something about indefinite pronouns. In 39th Annual Conference of the Cognitive Science Society (CogSci), 112-117.

Bernini, Giuliano \& Paolo Ramat. 1996. Negative Sentences in the Lan- 
guages of Europe: A Typological Approach. De Gruyter Mouton. doi:https://doi.org/10.1515/9783110819748.

Ferrer i Cancho, Ramon \& Ricard V. Solé. 2003. Least effort and the origins of scaling in human language. Proceedings of the National Academy of Sciences (PNAS) 100(3). 788-791. doi:https://doi.org/10.1073/pnas.0335980100.

Chierchia, Gennaro. 2013. Logic in Grammar: Polarity, Free choice, and Intervention. Oxford University Press. doi:10.1093/acprof:oso/9780199697977.001.0001.

Fauconnier, Gilles. 1975. Pragmatic scales and logical structure. Linguistic Inquiry 6(3). 353-375.

Frank, Michael C. \& Noah D. Goodman. 2012. Predicting pragmatic reasoning in language games. Science 336(6084). 998-998. doi:10.1126/science.1218633.

Haspelmath, Martin. 2001. Indefinite Pronouns. Oxford University Press. doi:10.1093/oso/9780198235606.001.0001.

Heim, Irene. 2006. Remarks on comparative clauses as generalized quantifiers. Unpublished manuscript, MIT.

Jacobs, Joachim. 1980. Lexical decomposition in Montague-grammar. Theoretical Linguistics 7(1-3). 121-136. doi:10.1515/thli.1980.7.1-3.121.

Jäger, Agnes. 2010. Anything is nothing is something. Natural Language \& Linguistic Theory 28(4). 787-822. doi:10.1007/s11049-010-9113-1.

Karttunen, Lauri \& Stanley Peters. 1979. Conventional lmplicature. In Choon-Kyu Oh \& David A. Dinneen (eds.), Syntax and Semantics 11: Presupposition, 1-56. New York: Academic Press. doi:10.1163/9789004368880_002.

Kemp, Charles \& Terry Regier. 2012. Kinship categories across languages reflect general communicative principles. Science 336(6084). 1049-1054. doi:10.1126/science.1218811.

Kemp, Charles, Yang Xu \& Terry Regier. 2018. Semantic typology and efficient communication. Annual Review of Linguistics 4. 109-128. doi:10.1146/annurevlinguistics-011817-045406.

Krifka, Manfred. 1994. The semantics and pragmatics of weak and strong polarity items in assertions. In Semantics and Linguistic Theory (SALT), vol. 4, 195-219. doi:10.3765/salt.v4i0.2462.

Ladusaw, William A. 1979. Polarity Sensitivity as Inherent Scope Relations: University of Texas, Austin PhD dissertation.

Lee, Young-Suk \& Laurence Horn. 1994. Any as indefinite plus even. Unpublished manuscript, Yale University.

Penka, Doris. 2011. Negative Indefinites. Oxford University Press. doi:10.1093/acprof:oso/9780199567263.001.0001.

Pereltsvaig, Asya. 2004. Negative polarity items in Russian and the 'Bagel problem'. In Sue Brown \& Adam Przepiorkowski (eds.), Negation in Slavic, 1-28. 
Complexity/informativeness trade-off in the domain of indefinite pronouns

Bloomington: Slavica Publishers.

Regier, Terry, Charles Kemp \& Paul Kay. 2015. Word meanings across languages support efficient communication. In Brian MacWhinney \& William O'Grady (eds.), The Handbook of Language Emergence, vol. 87, 237-263. Hoboken, NJ: Wiley-Blackwell. doi:10.1002/9781118346136.ch11.

Roberts, Ian \& Anna Roussou. 2003. Syntactic Change: A Minimalist Approach to Grammaticalization. Cambridge University Press. doi:10.1017/cbo9780511486326.

Rosch, Eleanor. 1978. Principles of categorization. In Eleanor Rosch \& Barbara Bloom Lloyd (eds.), Cognition and categorization, Hillsdale, NJ: Lawrence Erlbaum Associates.

Skyrms, Brian. 2010. Signals: Evolution, learning, and information. Oxford University Press. doi:10.1093/acprof:oso/9780199580828.001.0001.

Steinert-Threlkeld, Shane. 2019. Quantifiers in natural language optimize the simplicity/informativeness trade-off. In Amsterdam Colloquium 2019, http://events.illc.uva.nl/AC/AC2019/uploaded_files/inlineitem/ Steinert-Threlkeld_Quantifiers_in_natural_language_.pdf.

Uegaki, Wataru. 2020. NAND and the communicative efficiency model. Unpublished manuscript, University of Edinburgh.

Xu, Yang, Emmy Liu \& Terry Regier. 2020. Numeral systems across languages support efficient communication: From approximate numerosity to recursion. Open Mind 4. 57-70. doi:10.1162/opmi_a_00034.

Xu, Yang \& Terry Regier. 2014. Numeral systems across languages support efficient communication: From approximate numerosity to recursion. In 36th Annual Meeting of the Cognitive Science Society (CogSci), 1802-1807.

Xu, Yang, Terry Regier \& Barbara C. Malt. 2016. Historical semantic chaining and efficient communication: The case of container names. Cognitive Science 40(8). 2081-2094. doi:10.1111/cogs.12312.

Zeijlstra, Hedde. 2004. Sentential Negation and Negative Concord: University of Amsterdam $\mathrm{PhD}$ dissertation.

Zipf, George Kingsley. 1949. Human Behavior and the Principle of Least Effort. Cambridge, MA: Addison-Wesley. 
Denić, Steinert-Threlkeld and Szymanik

Milica Denić

Department of Linguistics

Institute for Logic, Language and Computation

University of Amsterdam

P. C. Hoofthuis, Spuistraat 134

1012 VB Amsterdam

The Netherlands

m.denic@uva.nl

Jakub Szymanik

Department of Linguistics

Institute for Logic, Language and Computation

University of Amsterdam

P. C. Hoofthuis, Spuistraat 134

1012 VB Amsterdam

The Netherlands

jakub.szymanik@gmail.com
Shane Steinert-Threlkeld

Department of Linguistics University of Washington

Guggenheim Hall, room 418D

Seattle, Washington 98195

USA

shanest@uw.edu 